\title{
Circumferential-Wave Dispersion Curves for Water-Immersed, Water-Filled Spherical Steel Shells
}

\author{
Michael F. Werby and Herbert Überall \\ Department of Physics, The Catholic University of America, Washington, DC 20064, USA
}

(Received 24 January 2003; accepted 17 April 2003)

\begin{abstract}
Frequency resonances in the scattering amplitude of acoustic waves from submerged elastic objects can be isolated by subtracting an underlying nonresonant background amplitude that is due to backscattered specular reflection. The resonances are caused by the phase matching of circumferential (surface) waves, and thus determine the phase-velocity dispersion curves of such waves. These are obtained here for the case of a spherical steel shell in water. If the shell is evacuated, Lamb-type waves $A_{0}$ and $S_{0}$ (analogous to those on a plate with one-sided water loading) are found, as well as the Scholte-Stoneley $(A)$ wave that propagates in the loading fluid. If the shell is filled with water, new circumferential waves propagating in the filler fluid appear and carry out a motion coupled with each other, causing drastic repulsion phenomena among their dispersion curves.
\end{abstract}

\section{INTRODUCTION}

Acoustic waves incident on an elastic shell cause the excitation of propagating waves on the shell, which may, in their totality, be referred to as "surface waves." If propagating over the curved portion of the shell, they may return to their point of origin and thus be referred to as "circumferential waves." If at a certain frequency, depending on the dispersion of these waves, they arrive at the point of origin with the same phase as that with which they left, their subsequent multiple circumnavigations lead to a resonant build-up of their amplitude. The corresponding resonances can be observed experimentally, ${ }^{1}$ as well as calculated from elasticity theory, ${ }^{2}$ the corresponding resonance frequencies are identical to the eigenfrequencies of the target.

Studies of the surface waves have previously been carried out for spherical shells ${ }^{2,3}$ that were immersed in a fluid as the acoustic medium, but that were evacuated (or air-filled; both in practice amounting to the same). For infinite cylindrical shells, however, the case of an internal fluid filling has been considered., ${ }^{4,5}$ It was found in this case that in the lowfrequency region, where one has the circumferential waves which are the analogues of the lowest-order Lamb waves on a plate $\left(A_{0}, S_{0}\right)$, one also has the fluid-borne Scholte-Stoneley wave $(A)$ resulting from the presence of the immersion fluid. In addition, there now exists a large variety of internal waves propagating circumferentially (or as breathing-type modes) in the filler fluid. Due to the fluid-elastic shell coupling, the fluid-borne and the shell-borne waves interact closely, causing what appears to be repulsion phenomena in the dispersion curves of these waves.

In the present investigation, the waves propagating internally in the filler fluid of a spherical steel shell are studied with regard to their resonances and their phase velocity dispersion curves, which follow one from the other; this study also obtains again the dispersion curves of the previously known waves on an evacuated, water-immersed shell. ${ }^{2,3}$ Similar extensive curve repulsion effects are found for the cylindrical shell, but with some differences. The analytic-numerical approach used here considers the individual partial wave amplitudes in the Rayleigh series of the backscattering ampli- tude. This permits a unique characterisation of the mode numbers and hence of the individual resonance values on the dispersion curves, as will be described below.

\section{CALCULATION OF PARTIAL-WAVE RESONANCES}

Phase matching of circumferential waves determines the relation between the resonance frequencies and the phasevelocity dispersion curves (the latter at the discrete resonance frequency points) for a spherical object $a s^{6}$

$$
c_{p} / c=(k a)^{*} /(n+1 / 2),
$$

(note that for circumferential waves on cylindrical objects, the term $1 / 2$ would be absent because of this being a twodimensional problem). Here, $c_{p}$ is the phase velocity of the surface wave, $c$ and $k$ are the sound speed and the wave number in the ambient fluid, respectively, with the asterisk denoting the value of $k$ at the resonance frequency, $a$ is the (outer) radius of the spherical object, and $n$ is the number of waves spanning the closed circumferential path, equivalent also to the partial wave number. There may exist a variety of surface waves that differ in their respective dispersion curves of $c_{p}$ plotted vs. the nondimensionalised frequency variable $k a$. Equation (1) makes it clear that in order to determine the curves of $c_{p} / c$ from the resonances one must know the value of $n$ at a given (measured or calculated) resonance point $(\mathrm{ka})^{*}$. Although special approaches have been employed towards this end, ${ }^{4}$ in the case of a calculational approach to the problem, it is straightforward to perform an individual evaluation of the partial-wave amplitudes corresponding to a given value of $n$, into which the total acoustic scattering amplitude is expanded in the form of a Rayleigh-type series. In this way, the resonances can be directly associated with the corresponding $n$ values. An illustration of the power of such an approach can be found in reference 2, where the resonances appearing at the coincidence frequency region of the evacuated-shell scattering amplitude could be identified uniquely, leading to the clarification of pseudo-Stoneley, or $A$ wave, resonances superimposed with the onset of the $A_{0}$ resonances. 